\title{
命HAD
}

DOI: http://doi.org/10.22585/hospdomic.v2i3.47

\section{Tendencias temporales de los patrones de búsqueda de información sobre cuidado domiciliario "Home Care" u hospitalario "Hospital Care" a través de Google}

Temporary trends in information search patterns about "Home Care" or hospital care "Hospital Care" through Google

María Sanz-Lorente ${ }^{1,2}$, Carmina Wanden-Berghe ${ }^{3,4}$

1. Centro de Salud Pública de la Consellería de Sanidad Universal y Salud Pública. Manises, España.

2. Universidad Miguel Hernández; Departamento de Salud Pública e Historia de la Ciencia. Sant Joant d'Alacant, Alicante, España.

3. Instituto de Investigación Sanitaria y Biomédica de Alicante (ISABIAL); Fundación para el Fomento de la Investigación Sanitaria y Biomédica de la Comunitat Valenciana (FISABIO), Alicante, España.

4. Hospital General Universitario, Alicante, España.

Correspondencia/Correspondence

María Sanz-Lorente

Centro de Salud Pública de la Consellería de

Sanidad Universal y Salud Pública. Manises,

España.

msanzlor@gmail.com

Recibido/Received

13.06.2018

Aceptado/Accepted

02.07 .2018
Conflicto de Intereses/Competing interest

Los autores no presentan conflicto de intereses.

CÓMO CITAR ESTE TRABAJO | HOW TO CITE THIS PAPER

Sanz-Lorente M. Wanden-Berghe C. Tendencias temporales de los patrones de búsqueda de información, sobre cuidado domiciliario "Home Care" u hospitalario "Hospital Care" a través de Google. Hosp Domic. 2018;2(3):93-9. 


\section{RESUMEN}

Objetivo: analizar las tendencias temporales de los patrones de búsqueda de información, sobre cuidado domiciliario "Home Care" u hospitalario "Hospital Care" a través de Google, desde el año 2004 a la actualidad.

Método: Estudio descriptivo transversal. Los datos se obtuvieron de la consulta directa a la herramienta "Google Trends». Términos de búsqueda "Home Care" y "Hospital Care". Fecha de consulta 10-05-2018.

Resultados: El volumen de búsqueda relativo $(\mathrm{VBR}=100)$ se alcanzó en enero de 2018 para "Home Care" y el menor en 2013 (VBR = 23,08) en el caso de "Hospital Care". El cociente medio entre ambos términos fue de 2,85, existiendo diferentes significativas entre los valores medios ( $\mathrm{t}$ de Student $=51,20$, gl 344, $\mathrm{p}<0,001$ ) . La evolución temporal del término "Home care" presentó un creciente ajuste a un modelo lineal ( $R 2=0,85$ y $p<0,001)$. En el caso de "Hospital care" la evolución fue ligeramente decreciente ( $R 2=0,19$ y $p<0,001)$. Los datos obtenidos y sus gráficos resultantes no mostraron ningún hito que merezca ser destacado.

Conclusiones: Se obtuvo un creciente volumen de búsqueda relativo con relación al término "Home Care", no así con "Hospital Care", sin la presencia de ningún hito destacable. Se considera que la semántica de búsqueda combinada puede ser en estudios sobre infoepidemiología, si bien serían necesarios más trabajos sobre la utilidad y limitaciones de esta herramienta.

Palabras clave: Servicios de Atención de Salud a Domicilio; Atención Hospitalaria; Gestión de la Información; Alfabetización Informacional; Medios de Comunicación Sociales.

\section{ABSTRACT}

Objective: To analyse the temporal trends in patterns of search for information about "Home Care" or "Hospital Care" via Google, since 2004 to the present day.

Method: Descriptive cross-sectional study. The data were obtained from the direct query to the Google Trends tool. Search terms "Home Care" and "Hospital Care". 10-05-2018 consultation date.

Results: The relative search volume (RSV = 100) was reached in January of 2018 for "Home Care" and the minor in $2013(\mathrm{RSV}=23.08)$ in the case of "Hospital Care". The ratio between both terms was 2.85, there were statistical differences between mean values ( $\mathrm{t}$ of Student = 51.20, gl 344, $\mathrm{p}<0.001$ ). The temporal evolution of the term "Home care" presented fit to a linear model (R2 $=0.85$ and $p<0.001)$. In the case of "Hospital care" evolution was slightly decreasing ( $R 2=0.19$ and $p<0.001)$. Data obtained and their resulting graphics did not show any landmark that deserves to be highlighted.

Conclusions: It was obtained a growing relative search volume in relation to the term "Home Care", not with "Hospital Care", without the presence of any noteworthy milestone. It is considered that the semantics of combined search may be in studies on infoepidemiology, but more works would be needed on the usefulness and limitations of this tool.

Keywords: Home Care Services; Hospital Care; Information Management; Information Literacy; Social Media. 


\title{
INTRODUCCIÓN
}

El cuidado domiciliario de la salud ha despertado gran interés y, afortunadamente, la adopción de la tecnología del «Internet de las cosas» está transformando la forma en que se proporciona la atención en el hogar, ofreciendo mejoras significativas para las personas que requieren cuidado, las familias que organizan la atención, así como para los profesionales que la brindan. En este contexto es interesante conocer la necesidad de información que la población general tiene sobre el cuidado domiciliario de la salud, esta información puede ayudar a mejorar los servicios y conocer las principales inquietudes.

La idea de que las poblaciones proporcionen datos sobre sus gustos e incluso sobre su enfermedad, a través del comportamiento de búsqueda de información en la Web, ya ha sido explorada en los últimos años (1). Existen trascendentes estudios que relacionan los datos obtenidos de las búsquedas con los casos de enfermedad, por ejemplo en la gripe (1,2), hepatitis (3) o VIH/SIDA (4).

Eysenbach (5), en el año 2009, acuñó el término de «Infoepidemiología» como un conjunto emergente de métodos de informática de salud pública para analizar el comportamiento de búsqueda, comunicación y publicación en Internet. Es decir, el seguimiento online de la información de salud (6).

Aunque, no es el útil más conocido de Google, "Google Trends» es una herramienta, de acceso libre, que informa del volumen total de búsquedas realizadas por los usuarios de todo el mundo para exponer qué tan frecuentemente se busca un término y en qué lugares. Básicamente, permite conocer cómo varía la notoriedad de los términos de búsqueda y comparar entre ellos. Esta herramienta muestra la popularidad relativa de los diferentes términos de búsqueda, a los que Google califica en una escala de 0 a 100 (este último representa el nivel más alto de búsquedas). Desafortunadamente, esto no aplica para todos los términos, ya que el nivel de búsquedas debe ser lo suficientemente representativo para aparecer en los resultados (7).

Se puede afirmar que «Google Trends» es una herramienta que facilita la investigación sobre las ciencias de la salud en la era de Big Data. Los datos de búsqueda en Internet pueden proporcionar información valiosa sobre los patrones de enfermedad y el comportamiento de la población (8).

En este contexto, este estudio tuvo como objetivo analizar las tendencias temporales de los patrones de búsqueda de información, sobre cuidado domiciliario "Home Care" u hospitalario "Hospital Care" a través de Google desde el año 2004 a la actualidad.

\section{MATERIAL Y MÉTODO}

\section{Diseño: Estudio descriptivo transversal.}

\author{
Fuente de obtención de la información:
}

Los datos se obtuvieron de la consulta directa, mediante acceso online, a la herramienta "Google Trends» (https://trends.google.es/trends/).

Como términos de búsqueda se utilizó: "Home Care" y "Hospital Care". No se pudo realizar más averiguaciones debido a que otras palabras clave no alcanzaban el umbral mínimo para obtener resultados, lo mismo que ocurrió con los términos en castellano. La fecha de consulta fue el 10 de mayo de 2018.

Los resultados proporcionados por la herramienta fueron descargados en formato normalizado comma-separated values (CSV) que permitió el almacenamiento posterior de los datos en un ar- 
chivo Excel. El control de la calidad de la información se efectuó mediante la doble entrada de los datos, corrigiendo las inconsistencias mediante la consulta con los originales. Para el análisis se utilizó el programa Statistical Package for the Social Sciences (SPSS) para Windows versión 21.0.

\section{Variables a estudio:}

Volumen de búsqueda relativo (VBR): Google Trends ofrece sus resultados desde un valor de 0 (es decir, volumen de búsqueda relativo inferior al 1\% del volumen máximo = umbral mínimo de visibilidad de resultados) a 100 (es decir, el volumen de búsqueda relativa alcanza su punto máximo). Por ejemplo, un volumen de búsqueda relativo de $50(\mathrm{VBR}=50)$ representa el $50 \%$ de la proporción de búsqueda observada más alta durante el período de estudio.

Evolución temporal: progreso de la tendencia de búsqueda a lo largo del tiempo (entre 2004 y la actualidad).

Vida media: mediana de la distribución del conjunto del volumen de búsqueda relativo ordenadas por su antigüedad.

Hito: acontecimiento puntual e importante que produce un cambio significativo en el volumen de búsqueda relativo (aumento o disminución brusca del número de búsquedas).

\section{Análisis de los datos:}

Para las variables cualitativas se calcularon las frecuencias absoluta y relativa (porcentaje) y las variables cuantitativas mediante la media y su desviación estándar, la mediana, el intervalo intercuartílico (IIQ), el máximo y mínimo.

Se analizó la existencia de asociación entre las variables cuantitativas para comprobar la significación de la diferencia de medias para muestras independientes, utilizando el test de la t de Student. La evolución temporal de las tendencias de búsqueda se examinó mediante el análisis de regresión, calculándose el coeficiente de determinación R2. El nivel de significación utilizado en todos los contrastes de hipótesis fue $a \leq 0,05$.

Para conocer la evolución del VBR se segmentó el período de estudio en dos etapas, tomando como punto de corte la vida media (la Mediana): $1^{a}$ época: de 2004 a 2011 y $2^{a}$ época: de 2012 a 2018.

\section{RESULTADOS}

A partir de las indagaciones realizadas se obtuvo la tendencia de búsqueda para ambos términos, observándose el interés según país; ver figura 1 (Los países que aparecen sin colorear se debe a que no alcanzaron el umbral mínimo para la obtención de resultados).

Los estadísticos, para todo el periodo analizado, del volumen de búsqueda relativo (VBR) para "Home Care" fueron: Media 71,41 \pm 0,89; Mediana 71; IIQ 61-79; Máximo 100 y Mínimo 49. Para "Hospital Care" se obtuvo: Media 25,06 0 0,17; Mediana 25; IIQ 24-27; Máximo 32 y Mínimo 20.

El VBR máximo (VBR $=100)$ se alcanzó en enero de 2018. El VBR medio mayor para "Home Care" se obtuvo en el año 2018 (VBR = 94,60), el menor en 2005 (VBR = 57,17). En el caso de "Hospital Care" el VBR medio mayor se consiguió en el año 2004 (VBR = 27,75) y el menor en 2013 $(\mathrm{VBR}=23,08)$. 
Figura 1. Resultados de las tendencias de búsqueda obtenidos en Google Trends mediante los términos "Home Care" y "Hospital Care" el 10 de mayo de 2018 y mapa, según interés de los diferentes países.
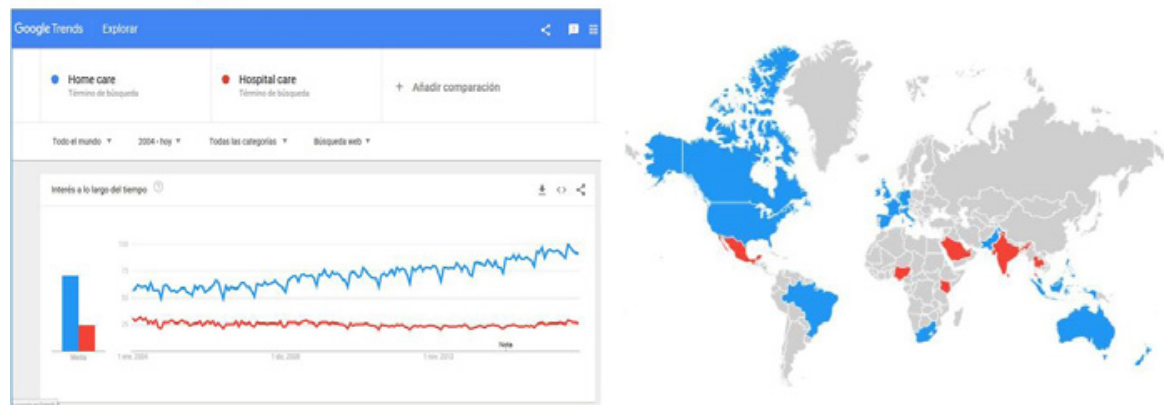

El cociente medio entre ambos términos fue de 2,85, existiendo diferencias significativas entre los valores medios (t de Student $=51,20$, gl 344, $p<0,001$ ).

La evolución temporal de las tendencias de búsqueda obtenidas en Google Trends mediante el término "Home care" presentó un gráfico con progreso creciente y ajuste a un modelo lineal (coeficiente de determinación R2 = 0,85 y p < 0,001). En el caso de "Hospital care" la evolución fue ligeramente decreciente con ajuste a un modelo lineal (coeficiente de determinación $R 2=0,19$ y $\mathrm{p}$ $<0,001)$; ver figura 2 .

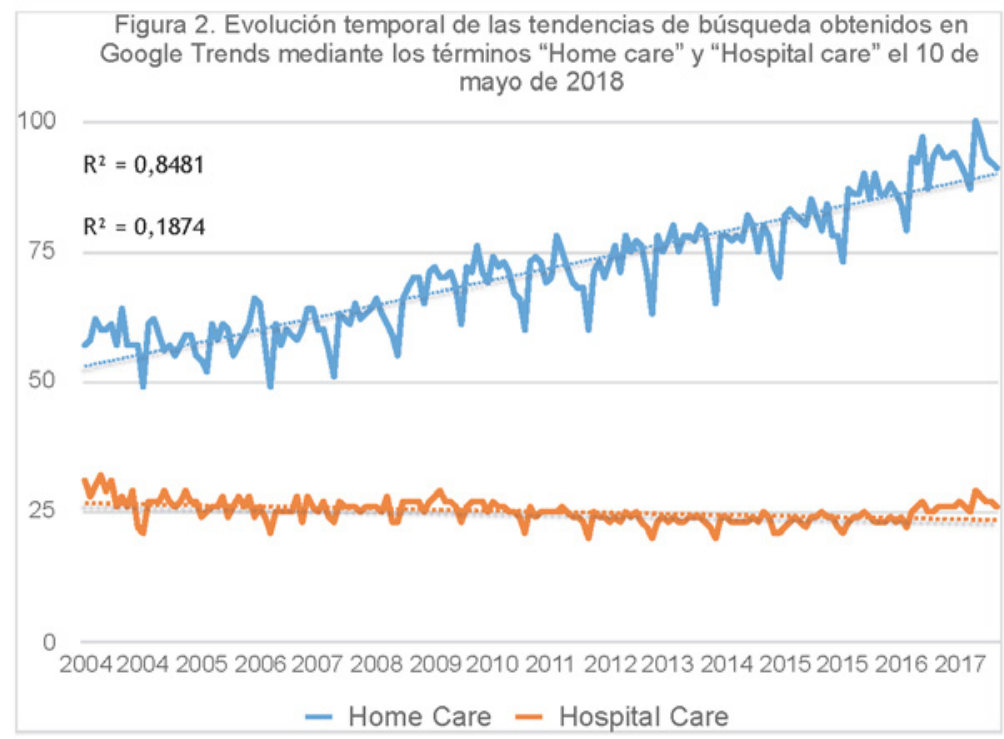

La vida media de la distribución del conjunto del VBR fue de 7 años. Para el término "Home Care" se observó que la media del VBR para la $2^{\mathrm{a}}$ época a estudio fue superior a la calculada para la $1^{\mathrm{a}}$ época: 81, 73 versus 63,14 (t de Student = -16,95; gl 171; $p<0,001$ ). Por el contrario, para "Hospital

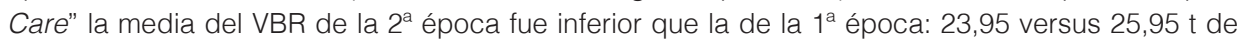
Student $=6,68 ;$ gl 171; $p<0,001$ ) 
Los datos obtenidos y sus gráficos resultantes no mostraron ningún hito que merezca ser destacado.

\section{DISCUSIÓN}

Como se ha comprobado, las tendencias de los motores de búsqueda son una herramienta innovadora para integrar la vigilancia, en tiempo real, de las necesidades de información sobre salud de la población.

Los datos del VBR obtenidos con «Google Trends» permitieron conocer el interés del público en los términos estudiados, quedando patente una mayor preocupación en la información sobre "Home Care".

La evolución temporal también favorece al término "Home Care", ya que su mayor coeficiente de determinación deja patente un mayor crecimiento del interés por el término. Por el contrario, en los resultados obtenidos con "Hospital Care" se aprecia una disminución de las búsquedas. Estas dos tendencias del patrón temporal, vienen también avaladas por las diferencias entre las dos épocas estudiadas. Mientras que en "Home Care" hay diferencias significativas del VBR a favor de la época más actual (crecimiento del interés a lo largo del tiempo), en "Hospital Care" es totalmente al contrario, es decir, un decrecimiento de las búsquedas en la época presente.

Por otro lado, la evolución temporal presenta una gráfica en diente de sierra (según estacionalidad) en la que no se puede reseñar ningún hito de especial interés. En otros estudios si se observó impactos apreciables motivados por brotes de la enfermedad o como respuesta a campañas de información puntuales que se traducían en una mejora de la búsqueda de información que podría derivar en estrategias efectivas y preventivas para el control de determinadas enfermedades (3).

La detección de picos de búsquedas relacionadas con enfermedad, es una destacada información para la vigilancia epidemiológica que ha demostrado correlación con los informes oficiales de varios países $(9,10)$.

Posibles limitaciones: de acuerdo con Johnson \& Mehta (7), no contar con datos de uso reales e intervalos de tiempo más precisos disminuye la capacidad de pronóstico. Además, se echa en falta una mayor transparencia, ya que no existe información sobre los métodos específicos que Google utiliza para pronosticar estas tendencias. Aparte, como sugieren Cervellin et al. (11), los resultados que se obtienen en «Google Trends» pueden estar influenciados por el interés de los medios. Y, se ha visto que los hitos se suelen alcanzar en un promedio de una a dos semanas después de que se publican noticias impactantes relacionadas con la salud (4).

Los resultados obtenidos pueden representar una nueva fuente de datos sobre la salud de la población, y aunque actualmente no se tenga en cuenta por los modelos de vigilancia de enfermedades, como advierten Tkachenko et al. (12), esta información puede ser utilizada de forma complementaria a los sistemas de vigilancia estándar.

Por todo lo observado, se podría concluir que se obtuvo un creciente volumen de búsqueda relativo con relación al término "Home Care", no así con "Hospital Care", sin la presencia de ningún hito destacable. Se considera que la semántica de búsqueda combinada puede ser en estudios sobre infoepidemiología, si bien serían necesarios más trabajos sobre la utilidad y limitaciones de esta herramienta. 


\section{BIBLIOGRAFÍA}

1. Pelat C, Turbelin C, Bar-Hen A, Flahault A, Valleron A-J. More diseases tracked by using Google Trends. Emerg Infect Dis. 2009;15(8):1327-8. DOI: 10.3201/eid1508.090299; PMID: 19751610

2. Valdivia A, Monge-Corella S. Diseases tracked by using Google trends, Spain. Emerg Infect Dis. 2010;16(1):168. DOI: 10.3201/eid1601.091308; PMID: 20031078

3. Ortiz-Martinez Y, Rios-González CM. Global impact of the World Hepatitis Day 2016: An evaluation using Google Trends. J Infect Public Health. 2017;10(5):690-1. DOI: 10.1016/j. jiph.2016.09.015; PMID: 28169192

4. Chiu APY, Lin Q, He D. News trends and web search query of HIV/AIDS in Hong Kong. PloS One. 2017;12(9):e0185004. DOI: 10.1371/journal.pone.0185004; PMID: 28922376

5. Eysenbach $\mathrm{G}$. Infodemiology and infoveillance: framework for an emerging set of public health informatics methods to analyze search, communication and publication behavior on the Internet. J Med Internet Res. 2009;11(1):e11. DOI: 10.2196/jmir.1157; PMID: 19329408

6. Eysenbach $\mathrm{G}$. Infodemiology and infoveillance tracking online health information and cyberbehavior for public health. Am J Prev Med. 2011;40(5 Suppl 2):S154-158. DOI: 10.1016/j.amepre.2011.02.006; PMID: 21521589

7. Johnson AK, Mehta SD. A comparison of Internet search trends and sexually transmitted infection rates using Google trends. Sex Transm Dis. 2014;41(1):61-3. DOI: 10.1097/ OLQ.0000000000000065; PMID: 24326584

8. Nuti SV, Wayda B, Ranasinghe I, Wang S, Dreyer RP, Chen SI, et al. The use of google trends in health care research: a systematic review. PloS One. 2014;9(10):e109583. DOI: 10.1371/journal. pone.0109583; PMID: 25337815

9. Chan EH, Sahai V, Conrad C, Brownstein JS. Using web search query data to monitor dengue epidemics: a new model for neglected tropical disease surveillance. PLoS Negl Trop Dis. 2011;5(5):e1206. DOI: 10.1371/journal.pntd.0001206; PMID: 21647308

10. Culquichicón-Sánchez C, Ramos-Cedano E, Chumbes-Aguirre D, Araujo-Chumacero M, Díaz Vélez C, Rodríguez-Morales AJ. Tecnologías de la Información y la Comunicación (TICs) en la vigilancia, prevención y control del dengue. Rev Chil Infectologia. 2015;32(3):363-4. DOI: 10.4067/S0716-10182015000400019; PMID: 26230448

11. Cervellin G, Comelli I, Lippi G. Is Google Trends a reliable tool for digital epidemiology? Insights from different clinical settings. J Epidemiol Glob Health. 2017;7(3):185-9. DOI: 10.1016/j. jegh.2017.06.001; PMID: 28756828

12. Tkachenko N, Chotvijit S, Gupta N, Bradley E, Gilks C, Guo W, et al. Google Trends can improve surveillance of Type 2 diabetes. Sci Rep. 2017;7(1):4993. DOI: 10.1038/s41598-017-05091-9; PMID: 28694479 